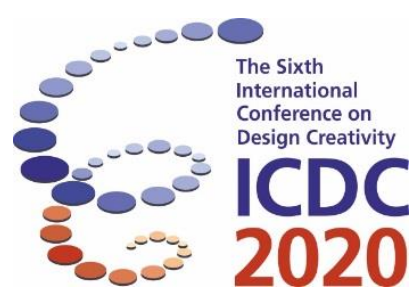

\title{
Success-oriented eco-ideation sessions: lessons learnt from the use of ten eco-design guidelines
}

\author{
Lorenzo Maccioni, Yuri Borgianni \\ Faculty of Science and Technology, Free University of Bozen-Bolzano, Bolzano, Italy
}

\begin{abstract}
The fields of eco-design and design creativity have not found strategic synergies yet. This applies despite the fact that the paramount objective of eco-design, i.e. sustainable development, might benefit from the radical design changes creativity can engender. In parallel, those significant changes should also support the transformation of products towards designs that exhibit major success chances, which is still in line with the perspectives of sustainable development. The authors have developed ten guidelines to guide eco-design towards creative and successful outcomes and the present paper illustrates the first experimentation thereof. The results of the experiment show that the compliance with the guidelines determines a satisfactory trade-off between environmental friendliness and success chances, as well as fully increasing the novelty of ideas. The outcomes are however affected by a remarked misalignment between the views of the two evaluators, i.e. an industrial player and an academic expert in eco-design.
\end{abstract}

Keywords: eco-ideation, success, design creativity, eco-design guidelines

\section{Context of the research and objectives}

The urgency of addressing ecological issues is nowadays of anecdotal evidence. Within design, the level of required changes is such that partial product modifications and redesigns can perhaps mitigate the ecological footprint, but cannot drive towards sustainable development (Vezzoli \& Manzini, 2008). It follows that, while radical innovation is required (Gaziulusoy \& Brezet, 2015), eco-design cannot overlook the contribution potentially provided by creativity and thinking out-of-the-box (Cucuzzella, 2016; Awan et al., 2019). On the other hand, while creativity is not totally neglected in eco-design (Sierra-Perez et al., 2016), the development has not taken place of bespoke and largely adopted methods and tools for leveraging creativity potential to trigger eco-innovation (Vallet et al., 2013). This conflicts with the evidence that the design and ideation of environmental friendly products is largely affected by methods (Faludi et al., 2019) and stimulation mechanisms (Tyl et al., 2018).

A potential key for blending eco-oriented and creative design endeavours can be found in the need to maximize the success chances of new designs, which is a prerogative of sustainable development ecodesign has not paid enough attention to so far (Skerlos, 2015). It is indeed a striking evidence that new environmental-friendly products have to replace previous product generations to pursue the intended eco-design objectives effectively (Maccioni et al., 2019a).

In this context, the authors have recently studied the effects of creative eco-oriented design endeavours in terms of success achievement (Maccioni et al., 2019a) and value creation (Maccioni et al., 2019b). The whole research has led to the definition of ten eco-design guidelines that are supposed to guide designers towards creative solutions that are viable to increase success chances. Details of the 
guidelines' development and extrapolation are omitted here, as this is out of the scope of the paper, and can be found in (Maccioni \& Borgianni, 2020). Indeed, the objective of the paper is to test the effects of coping with these guidelines in an eco-ideation session in terms of the expected outcomes (perspective success and environmental friendliness of ideas) and creativity metrics.

\section{Description of the eco-ideation session}

The present section specifies materials, methods and conditions in which the experiment to test the effectiveness of the ten eco-design guidelines was carried out. The experiment was followed by the evaluation of the generated ideas (Section 3).

\subsection{Participants}

28 Master students (participants hereinafter) were available to participate in an eco-ideation session. Those are Master students attending the module "Development and operation of product/servicesystems" (MSc. Engineering Design and Innovation) at Denmark Technical University (DTU). At the time of the experiment, they had already studied the eco-design principles proposed by Vezzoli \& Manzini (2008) and carried out projects on eco-design topics. Based on the modules' insights and amount of exercise, the participants can be considered as well trained in eco-design practice.

\subsection{Case Study}

A case study was defined based on the "Natura innovation challenge, zero waste packaging", i.e. an open innovation contest proposed by the Brazilian company Natura, whose aim is to reduce the environmental impact of their products (beauty products) http://innovationchallenge.natura/. The case study was presented and some existing products, solutions and product categories were shown through a picture taken from the above website to explain the most relevant development results achieved so far.

\subsection{Materials and experimental conditions}

The ten guidelines were introduced to participants in a twenty-minute presentation using illustrative products as implementation examples. A printed version of the ten guidelines and the appendix A of the book "Design for environmental sustainability" by Vezzoli \& Manzini (2008) were provided to the participants. Therefore, the authors opted to provide all the participants with both the guidelines proposed by Vezzoli \& Manzini (2008), as a reminder and a traditional reference for eco-design, and the ten guidelines (in the same form of the numbered list below) as a new complement to the former.

1. In eco-design, prioritize the optimization of the product lifecycle. Indeed, the understanding of the product scenario clarifies the design process that follows and drives towards acceptable solutions. Before implementing any strategy, you should:

- Consider every possible scenario that contextualizes the solution in a Circular Economy or Product-Service System context.

- Include functionalities in the same solution delivering additional value. To the scope, it is worth considering the functions of similar systems (TV broadcasts and the Internet), surrounding systems (the computer and the monitor) and systems performing opposite functions (the pencil and the eraser).

- Evaluate different Fields and Behaviours (mechanical, electric, magnetic, etc.) through which the function(s) can be performed.

2. Before trying to make a product more reliable through modular architectures (providing the possibility to replace components that will be damaged) or through integral architectures (reducing the number of components that can be damaged), explore different physical functioning principles.

3. Solutions exploiting new behaviours, fields or working principles are always perceived innovative but not sufficiently mature to be adopted. Understand what (structural, aesthetic, perceptual) features characterize traditional products and reproduce/copy them in the new solutions in order to increase the perception of familiarity. 
4. Develop self-repairing products or products that do not require maintenance at all. If the solution has to foresee repairing and/or maintaining during its life, introduce services that minimize the customer's involvement.

5. When you change the product's substance (solid, liquid, gas) to reduce the environmental impact of its packaging or transportation, stress the reason beyond this kind of solution and try to promote new benefits from this radical change in order to capture specific market sectors.

6. Avoid solutions that jeopardize the speed of delivery of the product to the customer and the safety during transport/distribution. When possible, use and distribute local products or components.

7. Do not limit your product's competitive advantage to a marginal improvement in terms of energy efficiency or material consumption.

8. Evaluate the chance of reducing resources toxicity and harmfulness, especially if consumers consider the product's material and behaviour as such. In any case, avoid increasing toxicity and harmfulness, although these are counterbalanced by different lifecycle advantages.

9. Do not neglect the quality-price ratio when you aim to resources' renewability and biocompatibility.

10. Implement actions that improve (the perception of) environmental sustainability during the use phase irrespective of the most critical product lifecycle stage.

The authors considered appropriate not to split the participants in two groups (control group and test group) for two main reasons.

- The overall restricted number of participants could lead to conclusions with poor explanatory power (effects mostly due to chance or people's creativity rather than the treatment).

- It could not be controlled whether participants adhered to the guidelines during the ideation session. In previous experiences of the authors' research group, participants have entrusted their own creativity or more established methods during creative sessions. This could have taken place also in the experimental situation, as the time to present the guidelines and suggest their use through appropriate tutorials was limited due to organizational reasons. In addition, the motivation of the participants could be not fostered by any means (surely, the time was insufficient to develop an idea up to the level requested in the open innovation contest) and this might have affected the quality of results (Battistella \& Nonino, 2012). Therefore, it was decided to evaluate ideas' compliance with guidelines (see Section 3) instead of distinguishing participants in different groups and evaluating the effect of a different treatment.

The participants were provided with the following instructions.

- You have to re-design (individually) products that have been showed and draft as many ideas as possible.

- Ideas have to be, from your viewpoint, viable, valuable and acceptable by customers/stakeholders.

- Ideas have to exhibit environment-related improvements; packaging-related improvements are particularly welcomed

- You can drastically change the solution and its context.

- Any idea has to be described in a written form, which can be accompanied by a drawing or sketch when applicable and useful to comprehension.

- It is fundamental that, for each idea, new competing factors, new benefits, new product attributes, and valuable effects for consumers and for the environment clearly emerge.

- A new idea can be expressed at any level of abstraction.

In addition, in order to communicate participants' ideas, a paper-based template to fill in was provided and explained. An example of an idea is shown in Figure 1. In the figure, it is possible to notice the three sections of the template. The first one is dedicated to describe textually the ideas (mandatory); in the example, it is possible to notice the consistency with the instructions mentioned above. The second one is optional and allowed participants to sketch the ideas when applicable and useful for explanation purposes. Also the third section is optional and it was meant to adding notes and variants for a full idea description. In this way, it was possible to collect all ideas in the same format and facilitate the subsequent evaluation task. 
Participants were assigned 45 minutes to generate ideas; during the ideation session, they were not left free to talk each other or to use electronic devices. Overall, 92 ideas have been generated by the participants (average 3.29, SD 1.22).

Idea-written description (Mandatory)
in order to reduce the environmental impact of the fragrances' packaging it is possible to
produce solid perfumes instead of liquid ones. In this way, glass is not needed and it can be
packaged with biomaterials like paper or recycled materials like steel or tin (that requires
cheaper production process). Moreover, steel and tin can be refilled or reused with different
scopes. In addition, the solid perfumes can be carried in a hand luggage on plane and its
packaging is less fragile. It could be possible to insert in the box additional hygiene tools e.g.
sustainable Q-Tips.

Sketch - when applicable and useful for the comprehension (Optional)

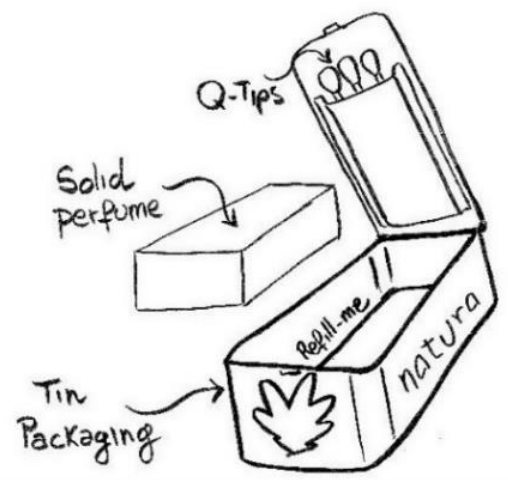

Notes and Variants (Optional)

- Round shapes

- Small thicknesses to be carried in pockets

Figure 1. Example of an idea communicated through the provided format

\section{Ideas evaluation and data elaboration}

\subsection{Metrics and variables characterizing the experiment}

To the scopes of the evaluation, the authors first judged every idea according to the consistency or the conflict with the ten guidelines. The variable Consistency was then created and assigned the value:

- 1 when the idea generated was deemed consistent with what the ten guidelines indicate -39 out of 92 ideas fulfilled this condition;

- 0 when no particular analogies or conflicts were found between the idea and the ten guidelines -38 out of 92 ideas;

- -1 when the idea contradicted one or more guidelines without complying with any of them -15 out of 92 ideas.

Second, a digital format of all the 92 ideas was created by transcribing the text fields and scanning the sketches. After that, the list of ideas in a digital format was sent to two experts, who, were, of course, unaware of the authors' consistency assessment.

- An industrial player expert in product development (Professional designer within the R\&D team of Natura);

- An academic expert in design for sustainability (Associate Professor doing research in ecodesign and Circular Economy at DTU). 
In line with the objectives of the paper, the experts were required to evaluate ideas' potential contribution to environmental sustainability and success. In particular, the industrial player can be considered one of the most reliable subjects in assessing the success chances of these ideas. As for the academic expert, given her recognized experience in sustainability and eco-design, she was primarily asked to evaluate each idea in terms of environmental sustainability. In addition to environmental sustainability and success, creativity was also assessed, as suggested by (Tyl et al., 2014). The traditional metrics used to assess ideation in (engineering) design creativity are Quantity, Variety, Novelty, Quality and Technical Feasibility (Shah et al., 2003). Those metrics are also applicable to assess the effectiveness of an ecoideation process, as confirmed by and López-Forniés et al. (2017) among others. The scholars proposed to use Novelty (in terms of something unusual or unexpected), Usefulness (in terms of applicability), technical feasibility and an environment-related metric (in terms of LCA estimates).

However, for this experiment, Quantity and Variety cannot be measured, make little sense, and/or are not thought as fundamental dimensions. As for the former, there are no separate samples or similar experiences to compare against - general indications about the volume of generated ideas are provided in Section 2.3. With regard to the latter, there are no established taxonomies to use when it comes to design ideation where no technical problem is explicated and generally in Product Planning (Bacciotti et al., 2016). In addition, environmental metrics cannot be quantified in terms of LCA results since the ideas have not achieved a sufficient level of detail. As a consequence, the environmental metric will be evaluated just in terms of perceived contribution to environmental sustainability by the recalled academic expert. Thus, this metric, along with all the other assessment criteria, will be evaluated subjectively by the involved experts.

The industrial expert was asked to evaluate his level of agreement with the following statements through a 6-point Likert scale (0 to 5).

- The idea is viable to achieve Success in the market - variable Success;

- I consider the idea Novel and/or Original - variable Novelty_ind;

- The idea is described in a comprehensive way and therefore it is featured by high Quality variable Quality_ind;

- The idea can be easily developed without particular technical problems. It is featured by high Technical Feasibility - variable Feasibility_ind.

The academic expert was asked to evaluate her level of agreement with the following statements through the same Likert scale.

- The idea presents clear advantages in terms of Environmental-Sustainability - variable Sustainability;

- I consider the idea Novel and/or Original - variable Novelty_aca;

- The idea is described in a comprehensive way and therefore it is featured by high Quality variable Quality_aca;

- The idea can be easily developed without particular technical problems. It is featured by high Technical Feasibility - variable Feasibility_aca.

It is possible to notice that the variables Novelty, Quality and Feasibility were assessed by both the evaluators since both of them have experience in the design field. The suffices _ind and _aca were clearly added to indicate that the evaluations were performed by one of the two experts.

A new variable that takes both success and sustainability into account was created by multiplying the values given by the academic expert in terms of Sustainability and the answers of the industrial player in terms of Success. A similar procedure was followed also in López-Forniés et al., (2017), in which the evaluations of the objective variables have been multiplied to obtain a single variable capable of indicating the solution that, overall, best meets ideation objectives. This new fictitious variable was named SustSucc and it features the ultimate objective of the guidelines since it represents the trade-off between success and sustainability. Indeed, SustSucc is 0 when either Success or Sustainability was assigned the value 0 and the maximum value when both Success and Sustainability got the highest score.

\subsection{Analysis and results of the evaluations}

At this point, each idea was characterized by a value for its consistency with the guidelines and multiple metrics featuring sustainability, success chances and creativity. 
At first, a regression between Consistency (independent variable) and SustSucc (dependent variable) was performed through the statistical software Stata (used in all the subsequent statistical analyses too). Results show that there is a significant relationship between the two (p-value $=0.043$ ) and the effect of compliancy with guidelines is positive (coefficient $=1.06$ ). This result can be considered essential for the preliminary verification of the expected effect of the guidelines in a creative eco-design process.

Subsequently, the creativity dimensions evaluated by both the two players (i.e. Novelty, Quality and Feasibility) were analysed. An Interrater Reliability analysis was performed to understand the level of agreement between the academic expert and the industrial player in evaluating the same ideas (results can be found in Table 1). The results show a basically absent agreement between the evaluators and therefore each variable has to be better considered independently as representative of a different phenomenon. As it could be hypothesized that the evaluators used preferentially different sections of the scale and/or perceived differently the ideas, a Spearman correlation was performed to understand if all the variables are correlated with monotonic relationships; the results are presented in Table 2.

Table 1. Interrater agreement between the academic expert and the industrial player

\begin{tabular}{|c|c|c|}
\hline Variable & Percentage of Agreement between the academic expert and the industrial player & Kappa \\
\hline Novelty & $10.99 \%$ & 0.0003 \\
\hline Quality & $10.99 \%$ & 0.0152 \\
\hline Feasibility & $17.58 \%$ & -0.0095 \\
\hline
\end{tabular}

Table 2. Spearman's rank correlation coefficients between evaluated variables

\begin{tabular}{|c|c|c|c|c|c|c|c|}
\hline & Success & $\begin{array}{c}\text { Novelty } \\
\text { _ind }\end{array}$ & $\begin{array}{c}\text { Quality } \\
\text { ind }\end{array}$ & $\begin{array}{c}\text { Feasibility } \\
\text { ind }\end{array}$ & Sustainability & $\begin{array}{c}\text { Novelty } \\
\text { _aca }\end{array}$ & $\begin{array}{c}\text { Quality } \\
\text { _aca }\end{array}$ \\
\hline Novelty_ind & 0.211 & 1 & & & & & \\
\hline Quality_ind & 0.329 & 0.298 & 1 & & & & \\
\hline Feasibility_ind & -0.261 & -0.505 & 0.045 & 1 & & & \\
\hline Sustainability & 0.052 & -0.044 & 0.150 & 0.154 & 1 & & \\
\hline Novelty_aca & 0.308 & 0.529 & 0.188 & -0.497 & 0.165 & 1 & \\
\hline Quality_aca & 0.086 & -0.032 & 0.191 & 0.118 & 0.623 & 0.419 & 1 \\
\hline Feasibility_aca & -0.254 & -0.229 & -0.065 & 0.134 & 0.178 & -0.213 & 0.345 \\
\hline
\end{tabular}

It is possible to notice that shared dimensions were evaluated differently by the industrial and the academic players. More specifically, the higher correlation in terms of the same dimension was achieved for Novelty (0.529) while a low agreement is shown for Quality (0.191) and Feasibility (0.134). It is interesting to notice that the highest correlation is between Quality_aca and Sustainability and that the coefficient is 0.623 (both the variables comes from the academician's evaluation), which leads to infer that quality and sustainability are somehow overlapping concepts in eco-design. Not surprisingly, negative correlations emerged between the Feasibility_ind with Novelty_ind (-0.505) and with Novelty_aca (-0.497). Moreover, it is interesting to notice how, for the academician, the concept of novelty and quality partially overlap (0.419) as well, while this does not apply to the same extent for the industrial player (0.298). This result could highlight differences in the appreciation of radical changes by the industrial and academic perspectives (the latter might be more prone to appreciate novel solutions).

Subsequently, ordered logistic regressions between all dimensions evaluated (dependent) and the Consistency (independent) were performed. Results can be found in Table 3.

In the table, it is possible to notice that no significant results emerged in terms of Quality. Conversely, significant outcomes can be found as for the effects on Novelty and Feasibility. More specifically, the effect of the consistency with the ten guidelines is positive in terms of Novelty and negative in terms of Feasibility. These are encouraging results, despite the negative indications about feasibility, as novelty is considered more relevant in this context because of the expectation of achieving non-incremental 
ideas. With regard to the effect of Consistency on Success and Sustainability, further considerations can be made for. First, the effect on Success is positive (although not significant within the acceptability range used as a rule of thumb) and this is an additional contribution to support the effectiveness of the guidelines. Second, even if the effect on Sustainability is not statistically significant (p-value >0.05), it seems evident that there is no risk to jeopardize sustainability by following the ten guidelines proposed. These outcomes support and specify the outcomes concerning the mixed variable that combines sustainability and success aspects.

Table 3. Results of the ordered logistic regressions linking assessments of the two evaluators and compliance with the eco-design guidelines. Positive coefficients (odd-ratios) indicate positive effects on the variables

\begin{tabular}{|c|c|c|c|c|c|}
\hline \multicolumn{3}{|c|}{ Industrial evaluator } & \multicolumn{3}{c|}{ Academic evaluator } \\
\hline Variable & Coefficient & P-value & Variable & Coefficient & P-value \\
\hline Success & 0.38 & 0.141 & Sustainability & 0.32 & 0.250 \\
\hline Novelty & 1.626 & 0.000 & Novelty & 1.1 & 0.000 \\
\hline Quality & 0.204 & 0.423 & Quality & -0.176 & 0.524 \\
\hline Feasibility & -1.07 & 0.000 & Feasibility & -0.802 & 0.004 \\
\hline
\end{tabular}

\section{Discussion and conclusions}

The present paper is mainly concerned with the verification of the goodness of a series of guidelines intended to support the creative development of successful and sustainable products. The guidelines have been developed based on previous literature and product evaluation experiments, thus they represent a theoretic construct and require validation. The first test has consisted in providing a number of design novices having a good experience in eco-design with the guidelines within an eco-ideation experiment. Based on the evaluation of the generated ideas, the compliance with the guidelines has determined a satisfactory trade-off between environmental friendliness and success chances. Moreover, an undisputable positive relationship has been found between the compliance with the guidelines and the novelty of ideas. The latter has been evaluated by both an industrial player (closely involved in the case study) and an academic expert in eco-design, who have provided contrasting views. However, said positive effect of the guidelines on novelty is confirmed for both the novelty evaluations considered separately. Differently, the compliance with the guidelines has played a negative role in terms of ideas feasibility - here, a dichotomy between novelty and feasibility is apparent, which is not unexpected in early design phases, as well as the two metrics are often seen as conflicting, e.g. (Starkey et al., 2016). Beyond the effectiveness of the guidelines, whose actual achievements will be better articulated below, the outcomes show that it is possible to achieve creativity, sustainability and creativity/novelty contextually in design. This supports the tenet of the present paper, i.e. a sustainability-oriented product development has to foresee radically new designs to face nowadays' environmental challenges without neglecting customer and market aspects. Clearly, the methodological development of instruments capable of targeting all the above objectives simultaneously is still in progress. While the guidelines used here represent a first effort in this direction, those cannot be considered fully validated because of the limitations of the present experiment, which follow and represent triggers for future studies. On the one hand, the compliance rather than the actual use of the guidelines has been tested because of the circumstances that have advised against the subdivision of participants into a control and a treatment group. It was deemed unlikely that a short exposition of the guidelines, which expectedly require some training and might benefit from changing the mind-set of (eco-)designers, could have affected the participants' way of designing and ideating considerably. Moreover, the generated ideas refer to a very early design phase and all the evaluations made concern the plausibility of these ideas to be novel, sustainable, successful, etc. Otherwise said, evaluators' individual sensibility might have greatly affected assessment and a larger number of evaluations would benefit the study. On the other hand, the guidelines should be articulated with respect to a traditional eco-design process. Indeed, they plainly refer to different design phases by addressing different hierarchies and aspects of products, from strategic lifecycle decisions to detailed design choices. Therefore, the guidelines should be integrated in 
a design process and more developments are required before testing them through an actual case study from industry. Here, it can be besides tested whether creative ideas will not be filtered out in the decision-making process, as a commonplace according to (Starkey et al., 2016), because of the emphasis on value and means to achieve success foreseen by the guidelines.

\section{Acknowledgement}

The study has been conducted in the framework of the project "fine-tuning new and smart ECO-design guidelines" (few sECOnds), funded by the Free University of Bozen-Bolzano. The authors wish to thank the volunteer students participating in the experiment, the company Natura for the use of the case study, the Denmark Technical University for making the conduction of the experiment possible, and the two evaluators for their valuable contribution to the present research.

\section{References}

Awan, U., Sroufe, R., \& Kraslawski, A. (2019). Creativity enables sustainable development: Supplier engagement as a boundary condition for the positive effect on green innovation. Journal of Cleaner Production, 226, 172-185. Bacciotti, D., Borgianni, Y., \& Rotini, F. (2016). A CAD tool to support idea generation in the product planning phase. Computer-Aided Design and Applications, 13(4), 490-502.

Battistella, C., \& Nonino, F. (2012). Open innovation web-based platforms: The impact of different forms of motivation on collaboration. Innovation, 14(4), 557-575.

Cucuzzella, C. (2016). Creativity, sustainable design and risk management. Journal of Cleaner Production, 135, 1548-1558.

Faludi, J., Ali, O., Srour, O., Mecanna, S., Kamareddine, R., \& Chatty, T. (2019). Preliminary Results Testing What Different Design Solutions Arise From Different Sustainable Design Methods. In Proceedings of the Design Society: International Conference on Engineering Design (Vol. 1, No. 1, pp. 3351-3360). Cambridge University Press.

Gaziulusoy, A. I., \& Brezet, H. (2015). Design for system innovations and transitions: a conceptual framework integrating insights from sustainablity science and theories of system innovations and transitions. Journal of Cleaner Production, 108, 558-568.

López-Forniés, I., Sierra-Pérez, J., Boschmonart-Rives, J., \& Gabarrell, X. (2017). Metric for measuring the effectiveness of an eco-ideation process. Journal of Cleaner Production, 162, 865-874.

Maccioni, L., \& Borgianni, Y. (2020). Bringing success and value in sustainable product development: the ecodesign guidelines. In Proceedings of the 7th International Conference on Sustainable Design and Manufacturing Maccioni, L., Borgianni, Y., \& Basso, D. (2019b). Value perception of green products: An exploratory study combining conscious answers and unconscious behavioral aspects. Sustainability, 11(5), 1226.

Maccioni, L., Borgianni, Y., \& Pigosso, D. C. (2019a). Can the choice of eco-design principles affect products' success?. Design Science, 5.

Shah, J. J., Smith, S. M., \& Vargas-Hernandez, N. (2003). Metrics for measuring ideation effectiveness. Design studies, 24(2), 111-134.

Sierra-Pérez, J., López-Forniés, I., Boschmonart-Rives, J., \& Gabarrell, X. (2016). Introducing eco-ideation and creativity techniques to increase and diversify the applications of eco-materials: The case of cork in the building sector. Journal of cleaner production, 137, 606-616.

Skerlos, S. J. (2015). Promoting effectiveness in sustainable design. Procedia CIRP, 29, 13-18.

Starkey, E., Toh, C. A., \& Miller, S. R. (2016). Abandoning creativity: The evolution of creative ideas in engineering design course projects. Design Studies, 47, 47-72.

Tyl, B., Legardeur, J., Millet, D., \& Vallet, F. (2014). A comparative study of ideation mechanisms used in ecoinnovation tools. Journal of Engineering Design, 25(10-12), 325-345.

Tyl, B., Vallet, F., \& Pialot, O. (2018). How to select appropriate stimulation mechanisms to perform an ecoideation session?. In DS 92: Proceedings of the DESIGN 2018 15th International Design Conference (pp. 27632774).

Vallet, F., Eynard, B., Millet, D., Mahut, S. G., Tyl, B., \& Bertoluci, G. (2013). Using eco-design tools: An overview of experts' practices. Design Studies, 34(3), 345-377.

Vezzoli, C., \& Manzini, E. (2008). Design for Environmental Sustainability. London, UK: Springer. 